\title{
2D-Cosy NMR Spectroscopy as a Quantitative Tool in Biological Matrix: Application to Cyclodextrins
}

\author{
Gilles Dufour, ${ }^{1,3}$ Brigitte Evrard, $^{1}$ and Pascal de Tullio ${ }^{2}$
}

Received 30 April 2015; accepted 14 July 2015; published online 25 August 2015

\begin{abstract}
Classical analytical quantifications in biological matrices require time-consuming sample pretreatments and extractions. Nuclear magnetic resonance (NMR) analysis does not require heavy sample treatments or extractions which therefore increases its accuracy in quantification. In this study, even if quantitative (q)NMR could not be applied to 2D spectra, we demonstrated that cross-correlations and diagonal peak intensities have a linear relationship with the analyzed pharmaceutical compound concentration. This work presents the validation process of a 2D-correlation spectroscopy (COSY) NMR quantification of 2-hydroxypropyl- $\beta$-cyclodextrin in plasma. Specificity, linearity, precision (repeatability and intermediate precision), trueness, limits of quantification (LOQs), and accuracy were used as validation criteria. $2 \mathrm{D}-\mathrm{NMR}$ could therefore be used as a valuable and accurate analytical technique for the quantification of pharmaceutical compounds, including hardly detectable compounds such as cyclodextrins or poloxamers, in complex biological matrices based on a calibration curve approach.
\end{abstract}

KEY WORDS: biological matrices; cyclodextrins; poloxamers; quantification; 2D-NMR.

\section{INTRODUCTION}

Besides the traditional use of nuclear magnetic resonance (NMR) spectroscopy as a structure elucidation tool, the interest for its quantification ability is continuously growing over years (1-3). Indeed, quantitative NMR (qNMR) is now widely used in various domains such as toxicology, metabolomics, pharmaceutics, or environmental $(4,5)$. In the pharmaceutical field, ${ }^{1} \mathrm{H}$ and ${ }^{13} \mathrm{C}$ NMR spectroscopies are often used for the identification and the quality evaluation of drugs such as busereline or gosereline or for some specific tests such as the determination of molar substitution of 2-hydroxypropyl- $\beta$-cyclodextrin (2,6). Despite the huge possibilities of qNMR regarding drugs quantification, the number of monographies in European pharmacopeia (EP) or US pharmacopeia (USP) is still limited. Under controlled conditions, ${ }^{1} \mathrm{H}$-NMR spectroscopy is considered as quantitative given that the intensity of a signal is directly related to the amount of resonant nuclei (7). However, in the case of complex solutions or mixtures (i.e., biological samples or pharmaceutical preparations ...), signal overlaps may occur and lead to poorly feasible quantification. Indeed, the

Brigitte Evrard and Pascal de Tullio equally supervised this work.

${ }^{1}$ Present Address: CHU, Laboratory of Pharmaceutical Technology and Biopharmacy, Department of Pharmacy, University of Liege, Tower 4, 2nd floor, Avenue de l'hôpital,1, 4000, Liège, Belgium.

${ }^{2}$ Laboratory of Medicinal Chemistry, Department of Pharmacy, Center for Interdisciplinary Research on Medicines (CIRM), University of Liege, 4000, Liège, Belgium.

${ }^{3}$ To whom correspondence should be addressed. (e-mail: gilles.dufour@ulg.ac.be) perfect integrations of overlapped signals, required to obtain accurate quantifications, could be complicated to asses, even if approaches such as deconvolution steps could be used (8). Two dimensional (2D) sequences such as homonuclear correlation spectroscopy $\left({ }^{1} \mathrm{H}_{-}{ }^{1} \mathrm{H}\right.$ COSY $)$ or ${ }^{1} \mathrm{H}_{-}{ }^{13} \mathrm{C}$ heteronuclear single-quantum correlation spectroscopy (HSQC) therefore constitute useful techniques given that the resolution can often be increased with the added dimension (1) as peaks are spread around along an additional orthogonal dimension (9). However, the direct quantitative properties observed in ${ }^{1} \mathrm{H}-\mathrm{NMR}$ cannot be extrapolated to 2D-NMR and especially in 2D-COSY. Indeed, cross-peak intensities are influenced by other factors than concentration such as relaxation times, mixing times, evolution times, uneven magnetization transfers, coupling constants $(\mathrm{J}) \ldots$ (10-12). Moreover, the influence of the biological matrix has to be taken into account and the presence of a huge amount of water requires the use of adapted pulse sequences including a pre-saturation step in order to drastically reduce the water signal which can greatly impact the quality of the spectra. However, even if qNMR could not be applied to 2DCOSY spectrum, it was expected that the cross-correlation and diagonal peak intensities have a linear relationship with the compound concentration. Indeed, several strategies have been developed to estimate the peak volume by getting rid of site-specific constant mainly influenced by relaxation times, pulse sequences delay... Among these strategies, the use of calibration curves or reference standards relies on more classical analytical techniques leading to high trueness and precision (9). We therefore decided to explore the use of 2DCOSY NMR spectroscopy for the identification and quantification of pharmaceutical excipients or drugs in complex 
biological media such as plasma, serum, or urine. According to the specificities of this spectroscopy and of the samples, a validation process is needed for highly accurate quantification. 2D-NMR presents several advantages including its ability to specifically detect a substance of interest in biological matrices without any sample pre-treatments (except the addition of $\mathrm{D}_{2} \mathrm{O}$ and TMSP) or sample extractions which decrease the potential errors due to experimenter manipulations encounter with traditional sample treatments. Moreover, NMR seems to be particularly adapted to the analysis of hardly detectable pharmaceutical compounds such as cyclodextrins and poloxamers for which limited quantification techniques are available and generally required long sample pre-treatments including extraction or proteins precipitation $(13,14)$. Taking into account these advantages, we decided to address this possible utilization of 2D-COSY spectroscopy for the identification and quantification of cyclodextrins in plasma medium. Pharmaceutical excipients have been considered for a long time as inert substances used to allow an efficient delivery of an active pharmaceutical ingredient (API). However, recent findings showed that some excipients are able to interact with other ingredients present in a formulation or cause adverse effects in patients and are therefore much more than a simple inert substance $(15,16)$. It appears that quantitative techniques are necessary to control their concentrations in blood following various administration routes to avoid accumulation leading to undesirable effects. Cyclodextrins (CDs) are cyclic oligosaccharides made up of linked $\alpha$-1,4-glucopyranose units which form a truncated cone-like structure comprising a hydrophobic cavity. Natural and derivative CDs, such as 2-hydroxypropyl- $\beta$ cyclodextrin (2-HP- $\beta-C D)$, are able to interact with poorly water-soluble drugs (BCS II and IV) in order to increase their apparent water solubility and therefore their oral bioavailability (17-19). Moreover, CDs can also be used as active ingredients in the treatment of pathologies such as Niemann-Pick disease (20) thanks to their abilities to interact with cholesterol. Besides cyclodextrins, we wanted to highlight the potential use of 2D-NMR in other pharmaceutical compound quantification. Among them, poloxamers are nonionic poly(ethylene oxide)-poly(propylene oxide) copolymers which are used in pharmaceutical preparations for their ability to act as surfactant, as solubilizing agents, as dispersing agents... Moreover, it has been shown that pluronic block copolymers are capable of sensitizing multi-drug resistant (MDR) cancer cells $(21,22)$ which highlight their biological activity. This work presents the possibilities of 2D-NMR techniques in the pharmaceutical field as a quantitative tool by describing the development and the full validation of a 2D-COSY quantification technique for 2-hydroxypropyl- $\beta$ cyclodextrin in plasma.

\section{MATERIALS AND METHODS}

\section{Chemicals and Solutions}

2-HP- $\beta$-CD (molar substitution $=0.64$ ) was kindly donated by Roquette (Lestrem, France). Poloxamers 188 and 407 were kindly donated by BASF (Ludwigshaven, Germany). Trimethylsilyl-3-propionic acid- $d 4$ (TMSP) and deuterium oxide $(99.96 \%$ D) were purchased from Eurisotop
(Gif-sur-Yvette, France). Certified maleic acid was obtained from Sigma-Aldrich (Karlsruhe, Germany). Ultrapure water (18.2 M $\Omega / \mathrm{cm}$ resistivity) was produced by a Milli-Q® system (Millipore). Pooled blank human plasma was purchased from the blood bank of the hospital (CHU, Liege).

\section{NMR Measurements}

All samples were recorded at $298 \mathrm{~K}$ on a Bruker Avance spectrometer operating at $500.13 \mathrm{MHz}$ for the proton signal acquisition. The instrument was equipped with a 5-mm TCI cryoprobe with a Z-gradient. Maleic acid was used as internal standard for quantification (1D experiment). Due to the nature of the samples, a presaturation sequence was used in all the experiments in order to minimize the water signal. All data were referenced to internal sodium 3-trimethylsilyl2,2,3,3-d4-propionate (TMSP) at $0.00 \mathrm{ppm}$ chemical shift (all spectra are calibrated with regard to TMSP). ${ }^{1} \mathrm{H}-\mathrm{NMR}$ spectra were acquired using a Carr-Purcell-Meiboom-Gill (CPMG) relaxation-editing sequence with presaturation. Upon the presence of proteins in plasma, the use of a sequence with a $\mathrm{T}_{2}$ filter (CPMG) greatly improves the baseline. The CPMG experiment used a RD-90-(t-180- $t) n$ sequence with a relaxation delay (RD) of $2 \mathrm{~s}$, a spin echo delay $(t)$ of $400 \mathrm{~s}$ and the number of loops $(n)$ equal to 80 . The water suppression pulse was placed during the relaxation delay (RD). The number of transients was typically 32 . The acquisition time was set to $3.983 \mathrm{~s}$ and a quantity of four dummy scans was chosen. The data were processed with the Bruker Topspin 2.1 software with a standard parameter set. The phase and baseline corrections were performed manually over the entire spectral range. Gradient-enhanced magnitude COSY experiment with a presaturation during relaxation delay was used for 2D measurements. Spectra were collected with 4096 points in $\mathrm{t} 2$ and 300 points in $\mathrm{t} 1$ over a sweep width of $10 \mathrm{ppm}$, with six scans per t1 value. The resulting COSY spectra were processed in Topspin 2.1 using standard methods, with zero-degree shifted sine-squared apodization in both dimensions and zero filling in $\mathrm{t} 1$ to yield a transformed 2D dataset of 2048 by 2048 points. It is well known that $2 \mathrm{D}$ peaks integration could drastically influence the volume determination and therefore the quantification process (23). In our experiments, peak volumes were determined by integration. To specifically quantify the peak of interest, we determined the integration region based on the highest concentration used in the validation process $(3.3 \mathrm{mM})$ and we checked in $3 \mathrm{D}$ that this region covered the entire peak volume. This integration region was therefore kept constant for all other concentrations in order to reach an accurate quantification process.

\section{Validation of the Method (2-HP- $\boldsymbol{\beta}-\mathrm{CD})$}

The validation of the method was performed in three series of experiments. The following criteria were tested: selectivity, linearity, precision (repeatability and intermediate precision), trueness, limits of quantification (LOQs), and accuracy. Total error was used as decision criterion for the validation process (24-26). The acceptance limits were set at $+/-15 \%$ (in accordance with FDA guidelines for bioanalyses) and the minimum probability to obtain future 
results within these limits was set at $\beta=90 \%$ ( $\beta$-expectation limits). Statistical analyses were performed using the e-noval ${ }^{\circledR}$ software (Arlenda, Liege, Belgium).

\section{Calibration Standards (2-HP- $\boldsymbol{\beta}-\mathrm{CD})$}

Stock solutions were individually prepared by dissolving $500 \mathrm{mg}$ of $2-\mathrm{HP}-\beta-\mathrm{CD}$ in $100 \mathrm{ml}$ of Milli-Q ${ }^{\circledR}$ water. A Mettler Toledo (Schwerzenbach, Switzerland) AT261 was used to weigh $2-\mathrm{HP}-\beta-\mathrm{CD}$ (precision: $10 \mu \mathrm{g}$ ). All weights were corrected for the water content after its measurement by Karl-Fischer titration (V30, Mettler Toledo). Calibration standards at four concentration levels within the range of $0.1-3.3 \mathrm{mM}(0.1,0.33,1.65$, and $3.3 \mathrm{mM})$ were obtained by dilution of the stock solution with Milli-Q ${ }^{\circledR}$ water. At $100 \mu \mathrm{l}$ of these solutions, $100 \mu \mathrm{l}$ of blank plasma, $50 \mu \mathrm{l}$ of $\mathrm{D}_{2} \mathrm{O}$ buffer, $50 \mu \mathrm{l}$ of maleic acid $(5 \mathrm{mM})$, and $5 \mu \mathrm{l}$ of TMSP were added for NMR analysis. Each concentration level was analyzed three times for three series of experiments (three independent days). Calibration curve on day 3 was realized with different human plasma in order to take into account the variable protein/peptide composition between different plasmas.

\section{Validation Standards (2-HP- $\beta$-CD)}

Validation standards were prepared in the same way as the calibration standards. Five concentrations $(0.1,0.165$, $0.33,1.65$, and $3.33 \mathrm{mM}$ ) were analyzed three times for three series of experiments (three independent days). Validation standards are considered as true values by consensus. Validation standards on day 3 were prepared with different human plasma in order to take into account the variable protein/peptide composition between different plasmas.

\section{Application of the Technique}

The generic method was applied to other pharmaceutical excipients. Solutions comprising either poloxamer 188 or 407 were prepared by dissolving $150 \mathrm{mg}$ in $5 \mathrm{ml}$ of Milli-Q ${ }^{\circledR}$ water. At $10 \mu \mathrm{l}$ of these solutions, $1000 \mu \mathrm{l}$ of blank plasma, $200 \mu \mathrm{l}$ of $\mathrm{D}_{2} \mathrm{O}$ buffer, $50 \mu \mathrm{l}$ of maleic acid $(5 \mathrm{mM})$, and $5 \mu \mathrm{l}$ of TMSP were added for NMR analysis. The validated method was applied to mice plasma quantification further to intraperitoneal (i.p.) injections. In vivo experiments were carried out in accordance with the local ethic committee rules on males BALB/c mice of 6 to 8 weeks old.

\section{RESULTS}

\section{Method Development}

The general aim of our research was the setting of a quantification method for hardly detectable pharmaceutical compounds in biological matrices without complicated extraction or separation steps in order to reach a high accurate quantification and that could be used in toxicological studies. We therefore decided to evaluate the interest of 2D-COSY spectroscopy for its ability to unambiguously identify substances of interest in biological matrices and for its absence of long sample pre-treatments. We firstly focused on the quantification of cyclodextrins and especially of 2hydroxypropyl- $\beta$-cyclodextrin $(2-\mathrm{HP}-\beta-\mathrm{CD})$. The first step in this development was the ${ }^{1} \mathrm{H}-\mathrm{NMR}$ analysis of $2-\mathrm{HP}-\beta-\mathrm{CD}$ in water to identify signals of interest. As previously shown (27), we decided to focus on the wider signal at $1.1 \mathrm{ppm}$ (Fig. 1b) which corresponds to the methyl of the hydroxypropyl group (Fig. 1a) in order to specifically detect $2-\mathrm{HP}-\beta-\mathrm{CD}$. Other signals (e.g. signal at $5 \mathrm{ppm}$ ) could also be used for the quantification of unsubstituted cyclodextrins (27). Given that overlapping signals corresponding to lipids and proteins of the plasma could be present in the 1.1-ppm spectral zone, the classical 1D NMR approach could not be applied for quantification. Indeed, even if deconvolution (total-lineshape) analysis could be used for the quantification of 2-HP$\beta-C D$ at high concentrations (minimal signal overlap), lower concentrations could not be accurately quantified given that the overlapping is too important (Fig. 2). We therefore decided to analyze the cross-peak interaction between the doublet at $1.1 \mathrm{ppm}$ and the central cavity of the cyclodextrin represented by the proton $\mathrm{H} 3(4 \mathrm{ppm})$ by using the $2 \mathrm{D}$ COSY approach. Thanks to the added dimension, lipids or proteins contained in the plasma do not influence the specific determination of 2-HP- $\beta-C D$ amount (Fig. 3). As previously explained, qNMR could not be applied to 2D-COSY data. Moreover, due to specificities of $2-\mathrm{HP}-\beta-\mathrm{CD}$, such as uncertainty in the molecular weight and in the substitution degree and the relative broadness of the NMR signals, the application of quantitative NMR (qNMR) could be difficult. We therefore undertook a validation process to identify the most appropriate linear regression model. It is important to note that the molar substitution of 2-HP- $\beta-C D$ influence signal intensities and integrations. Calibration curves must therefore be made with 2-HP- $\beta-C D$ coming from the same batch and with the same mean substitution degree as the cyclodextrin to analyze.

\section{Validation Method (2-HP- $\boldsymbol{\beta}-\mathrm{CD})$}

According to ICH Q2 (R1) guidelines (28), several validation criteria were evaluated as described in "Validation of the Method (2-HP- $\beta-C D)$ " section. An accuracy profile based on tolerance intervals was used to evaluate the reliability of the results (29). $\beta$-Expectation tolerance intervals defined an interval space in which it is expected that each future result will fall with a defined probability $(\beta)$. This tolerance interval is computed for each validation standards concentration levels on the basis of their estimated intermediate precision, standard deviation, and bias. The accuracy profile is defined by plotting the upper tolerance limit on one hand and the lower tolerance limit on the other hand. While this accuracy profile stays within the previously set acceptance limits, the method is considered as validated. This approach guarantees that each further measurement of unknown samples will be included within the tolerance limits with at least a probability of $\beta$, e.g., $90 \%$.

\section{Method Specificity}

From Fig. 1b, we could see different signals from 1.1 to $5.2 \mathrm{ppm}$. All these signals could be used for quantification. Given that the signal at $1.1 \mathrm{ppm}$ is specific to cyclodextrins 

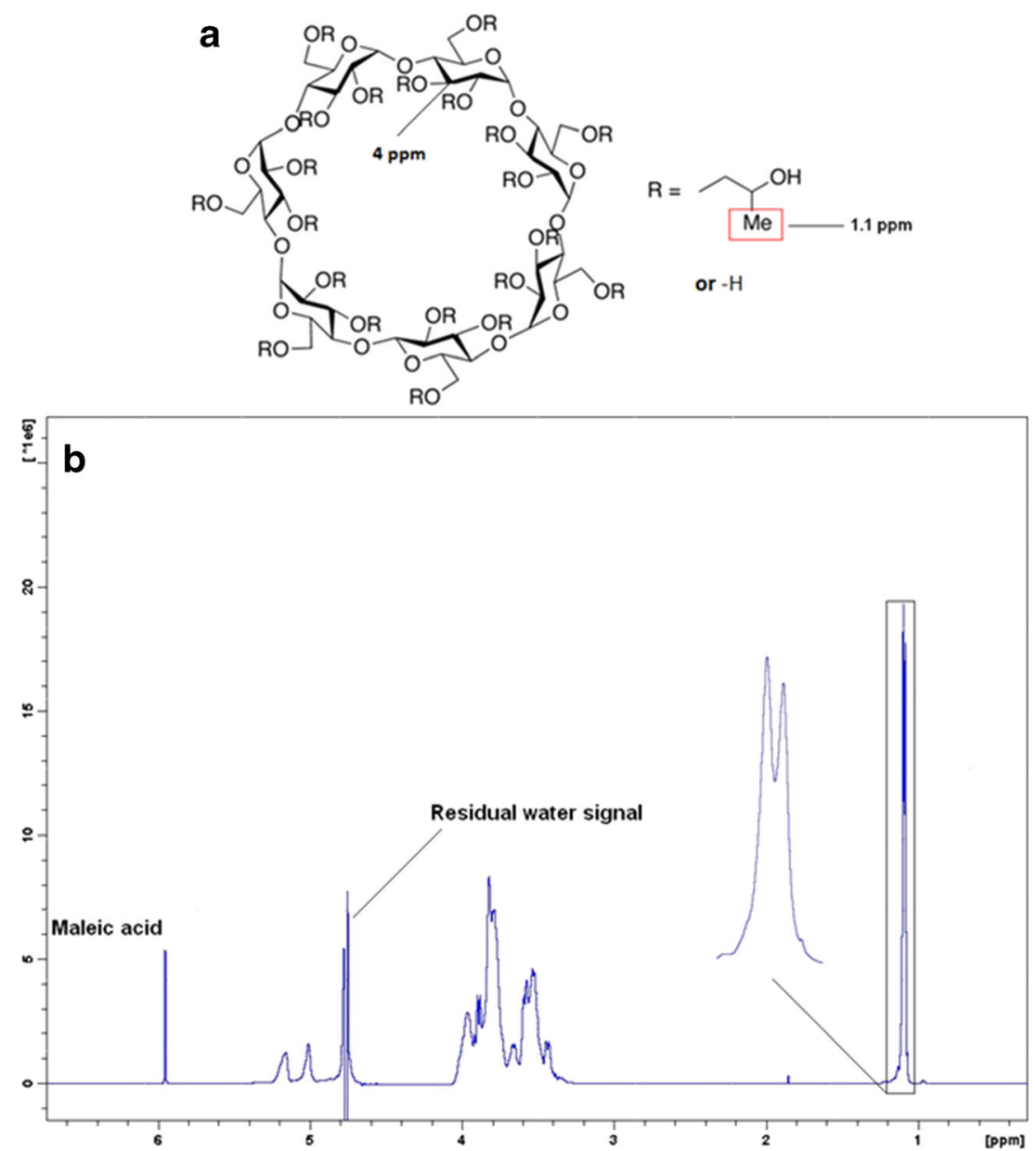

Fig. 1. Chemical structure of 2-hydroxypropyl- $\beta$-cyclodextrin; $R$ =hydroxypropyl (a) ${ }^{1} \mathrm{H}$ NMR spectrum of water solution spiked with 2-hydroxypropyl- $\beta$-cyclodextrin (d.s 0.64 ) (b). The signal at $1.1 \mathrm{ppm}$ corresponding to the methyl of the hydroxypropyl group is enlarged

possessing a hydroxypropyl group, we selected this doublet. The specificity compared to other compounds (lipids and proteins) present in the plasma was assessed by adding increasing concentration of $2-\mathrm{HP}-\beta-\mathrm{CD}$ into blank plasma. The signal corresponding to the methyl of the hydroxypropyl group at $1.1 \mathrm{ppm}$ can therefore be unambiguously identified in the plasma (Fig. 2). The 2D-COSY specificity is also ensured given that no other correlation cross-peaks have been detected in the zone of interest at 1.1/4 ppm (Fig. 3). In accordance with FDA bioanalytical method validation guidance, six individual blank plasma samples were used to demonstrate the absence of interference in the $1.1 / 4$ ppm area.

\section{Response Function}

The response function of an analytical method is, within the range, the existing relationship between the signal intensity and the concentration of the analyte in the sample. In order to determine the most accurate calibration curve, different models were applied. The calibration curves were built with four concentration levels within the range $0.1-3.3 \mathrm{mM}$. Validation standards were prepared in the same way at five concentration levels. Each of them was analyzed in triplicate on three series of experiment in order to evaluate inter- and intra-assay precision. By using different regression models, validation standards concentrations were back-calculated to determine a precision and a trueness index. On this basis, the regression model which provides the most appropriate response function in terms of accuracy, dosing range index, precision, and trueness is a linear regression after square root transformation. The calibration curve obtained from this regression model is defined by the following equation: $\operatorname{sqrt}(Y)=a+b \operatorname{sqrt}(X)$ where $Y$ is the analytical response and $X$ is the introduced concentration $(\mathrm{mM})$.

\section{Trueness}

Trueness refers to the closeness of agreement between a conventionally accepted value (reference value) and a mean experimental one. It therefore gives information on systematic error. According to the appropriate model (linear regression after square root transformation), validation standard concentrations were back-calculated to determine trueness expressed in terms of relative bias (\%) at each concentration level of the validation standards. As shown in Table I, trueness never exceeded $6.25 \%$. 

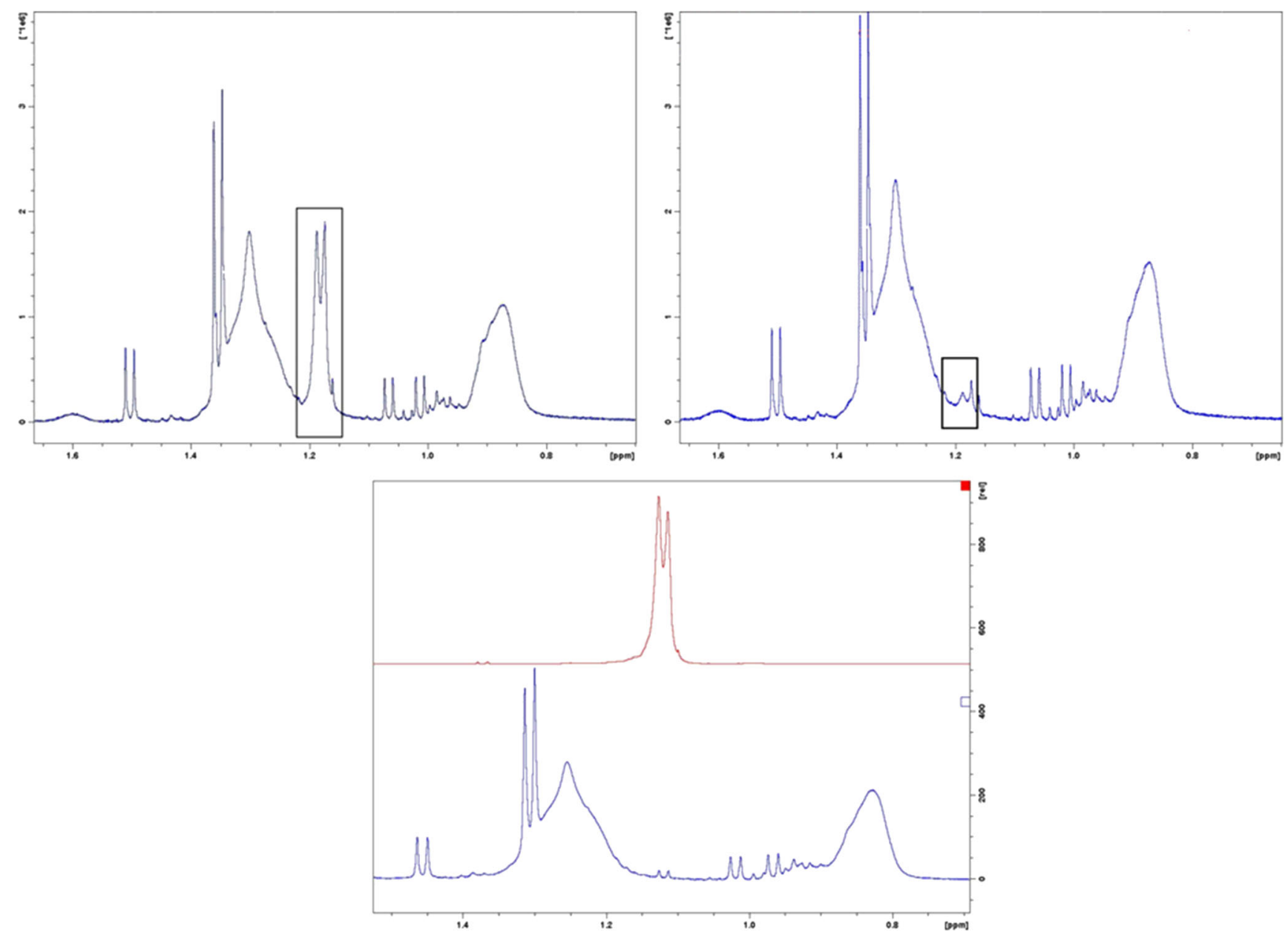

Fig. 2. ${ }^{1} \mathrm{H}-\mathrm{NMR}$ spectra of 2-hydroxypropyl- $\beta$-cyclodextrin in plasma at concentrations of $1.5 \mathrm{mM}$ (upper right) and $0.15 \mathrm{mM}$ (upper left). The signal at $1.1 \mathrm{ppm}$ corresponding to the methyl of the hydroxypropyl group is surrounded. Blank plasma spectrum focus on $1.1 \mathrm{ppm}$ area (blue) with the ${ }^{1} \mathrm{H}-\mathrm{NMR}$ spectrum of water solution spiked with 2 -hydroxypropyl- $\beta$-cyclodextrin ( red)

\section{Precision}

Precision is the closeness of agreement among measurements from multiple sampling of a same homogeneous sample. It therefore gives information on random errors and it can be evaluated at two levels: repeatability and intermediate precision. Precision is described in terms of relative standard deviation (RSD) values at each concentration level of the validation standards. Results are computed in Table I. Relative biases related to repeatability and intermediate
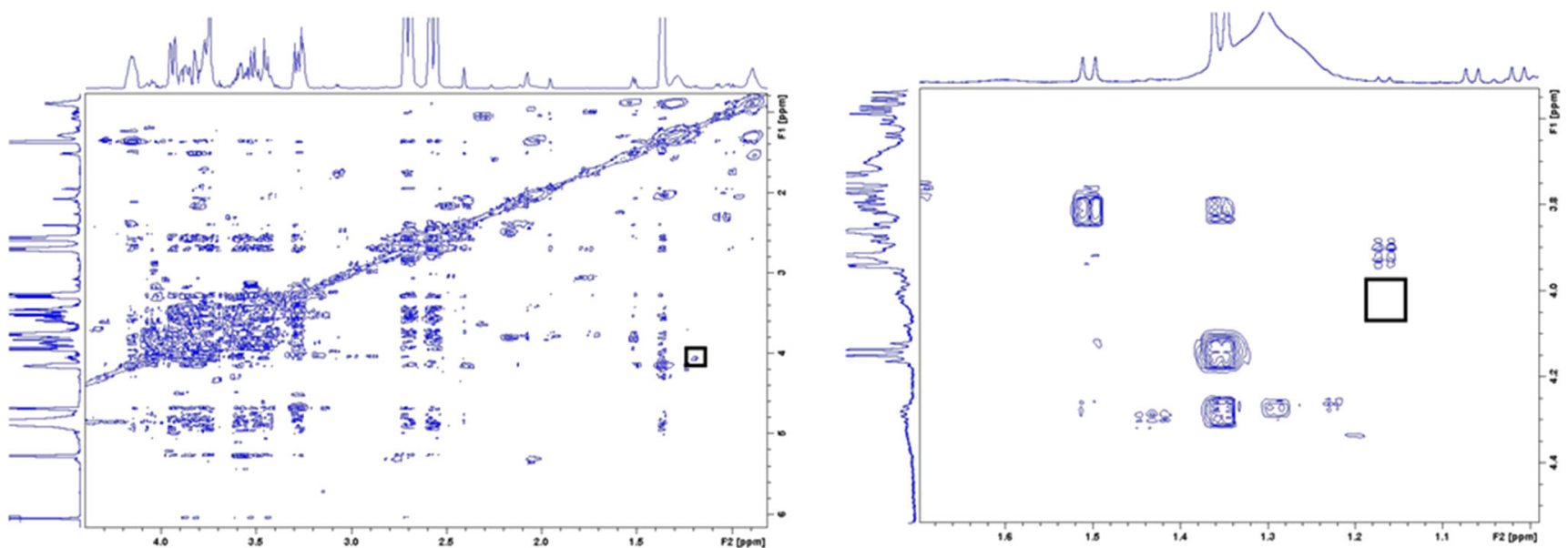

Fig. 3. $2 \mathrm{D}$-COSY NMR spectra of 2 -hydroxypropyl- $\beta$-cyclodextrin in plasma at a concentration of $0.112 \mathrm{mM}$ (left). The correlation spot between the methyl of the hydroxypropyl group $(1.1 \mathrm{ppm})$ and the proton $\mathrm{H} 3$ of the cyclodextrin central cavity $(4 \mathrm{ppm})$ is enlarged. Blank plasma spectrum focus on $1.1 / 4 \mathrm{ppm}$ area (right). A square highlight the absence of interference in this spectral zone 
Table I. Validation Criterion for 2D-COSY NMR Quantification of 2-HP- $\beta$-CD in Plasma by Using the Cross-Peak Interaction Between the Signal at $1.1 \mathrm{ppm}$ and the Signal at $4 \mathrm{ppm}$

Validation criteria

\begin{tabular}{|c|c|c|}
\hline $\begin{array}{l}\text { Response function } \\
\text { (calibration curve) }\end{array}$ & $\begin{array}{l}\text { Linear regression } \\
\text { after square } \\
\text { root transformation }\end{array}$ & \\
\hline \multicolumn{3}{|l|}{ Trueness } \\
\hline Level & Conc $\left(10^{-3} \mathrm{M}\right)$ & Rel bias (\%) \\
\hline Level 1 & 3.265 & -1.072 \\
\hline Level 2 & 1.753 & 6.243 \\
\hline Level 3 & 0.316 & -4.344 \\
\hline Level 4 & 0.156 & -5.742 \\
\hline Level 5 & 0.098 & -0.913 \\
\hline \multicolumn{3}{|l|}{ Precision } \\
\hline Level & Repeatability (RSD\%) & $\begin{array}{l}\text { Intermediate } \\
\text { precision }(\mathrm{RSD} \%)\end{array}$ \\
\hline Level 1 & 3.714 & 4.659 \\
\hline Level 2 & 2.464 & 2.962 \\
\hline Level 3 & 1.223 & 2.129 \\
\hline Level 4 & 2.66 & 2.663 \\
\hline Level 5 & 1.598 & 4.778 \\
\hline \multicolumn{3}{|l|}{ Accuracy } \\
\hline Level & $\begin{array}{l}\beta \text {-Expectation } \\
\text { tolerance limits (\%) }\end{array}$ & \\
\hline Level 1 & $-12.28 / 10.14$ & \\
\hline Level 2 & $-1.214 / 13.7$ & \\
\hline Level 3 & $-10.41 / 1.725$ & \\
\hline Level 4 & $-11.59 / 0.107$ & \\
\hline Level 5 & $-15.80 / 13.98$ & \\
\hline \multicolumn{3}{|l|}{ Linearity } \\
\hline Slope & 0.999 & \\
\hline Intercept & 0.0079 & \\
\hline $\mathrm{R}^{2}$ & 0.996 & \\
\hline
\end{tabular}

precision never exceeded $4.78 \%$ which confirms the high precision of the validated methods.

\section{Accuracy and Linearity}

Accuracy refers to the closeness of agreement between the test result and the accepted reference value (conventionally true value). The accuracy therefore takes into account the total error, i.e., systematic and random errors, related to the test result. Upper and lower bounds of $\beta$ expectation tolerance intervals at the $10 \%$ level were calculated at each concentration levels and did not exceed the previously set acceptance limits (15\%) between 0.112 and $3.33 \mathrm{mM}$ as shown in Fig. 4. This method is therefore considered as validated within this range. This means that the levels for which the $\beta$-expectation tolerance intervals obtained are outside the acceptance limits could not be measured with sufficient accuracy and therefore could not be included in the dosing range. The linearity of a method refers to its ability within a definite range to obtain results directly proportional to the concentration of the analyte in the sample. Linearity was observed in the working range of concentrations (Table I).

\section{$L O Q S$}

Limits of quantifications were obtained by calculating the smallest and the highest concentration beyond which the accuracy limits (or $\beta$-expectation limits) previously described go outside the acceptance limits $(+/-15 \%)$. The dosing range is therefore defined as the interval between the upper and lower limits where the analytical 2D-COSY method achieves adequate accuracy. As shown in Fig. 4, the method is considered as valid within the range for which the dashed curves (accuracy profile) are within the dotted acceptance limits and the lower limit of quantification (LLOQ) is therefore set at $0.112 \mathrm{mM}$. LLOQ could be lowered by using a higher scan number but related to the intended use of this quantification method, it is not necessary to reach smaller concentrations given that the toxic $2-\mathrm{HP}-\beta-\mathrm{CD}$ dose is above this LLOQ.

\section{Applications}

Next to this validation process, we analyzed plasma spiked with poloxamers in order to highlight the potential use of 2D-NMR in other pharmaceutical compound quantification. Some analytical methods have been reported for poloxamer quantification in pharmaceutical preparations (30-32), but despite their wide range of pharmaceutical applications and especially their recognized biological effect $(21,22)$, limited analytical techniques have been developed for their quantification in biological matrices. We therefore decided to evaluate the applicability of 2D-COSY spectroscopy as a quantitative technique for poloxamers 188 and 407 in plasma. We firstly focused on identifying a well-resolved signal of interest that could be used for the quantification by using ${ }^{1} \mathrm{H}-\mathrm{NMR}$. As shown in Fig. 5, the wider signal at $1.2 \mathrm{ppm}$ (displayed for poloxamer 188) corresponding to the methyl group could be used to that purpose. However, as for $2-\mathrm{HP}-\beta-\mathrm{CD}$, lipids and proteins present in the plasma impair its use (especially for low concentrations) in 1DqNMR (Fig. 6). We therefore decided to focus on the crosspeak interaction between this methyl signal at $1.2 \mathrm{ppm}$ and the $\mathrm{CH}_{2} \mathrm{O} / \mathrm{CHO}$ integrals between $\sim 3.6$ and $3.9 \mathrm{ppm}$ (Fig. 7). This intense correlation spot appears free of interference and could be used for the specific quantification of poloxamers in plasma by using the same approach developed for the quantification of $2-\mathrm{HP}-\beta-\mathrm{CD}$. Moreover, in order to assess the applicability of our technique to specifically detect 2-hydroxypropyl- $\beta$-cyclodextrin following a non i.v. route of administration, we performed in vivo study on mice. The aim of this study was to demonstrate the possible recovery and quantification of 2-hydroxypropyl- $\beta$ cyclodextrin in plasma following i.p. injections. Mice were therefore injected with various 2-hydroxypropyl- $\beta$ cyclodextrin concentrations (from 250 to $2000 \mathrm{mg} / \mathrm{kg}$ ), and $20 \mathrm{~min}$ later, their plasmas were recovered for quantifications. For all tested concentrations, we were able to detect and specifically quantify this cyclodextrin. All concentrations were above the LLOQ $(0.112 \mathrm{mM})$. This method could be therefore be used to monitor plasma concentration following various administration route in human or mice toxicological studies. Besides the quantification in biological fluids, 2DNMR could also be used to quantify 2-hydroxypropyl- $\beta$ - 

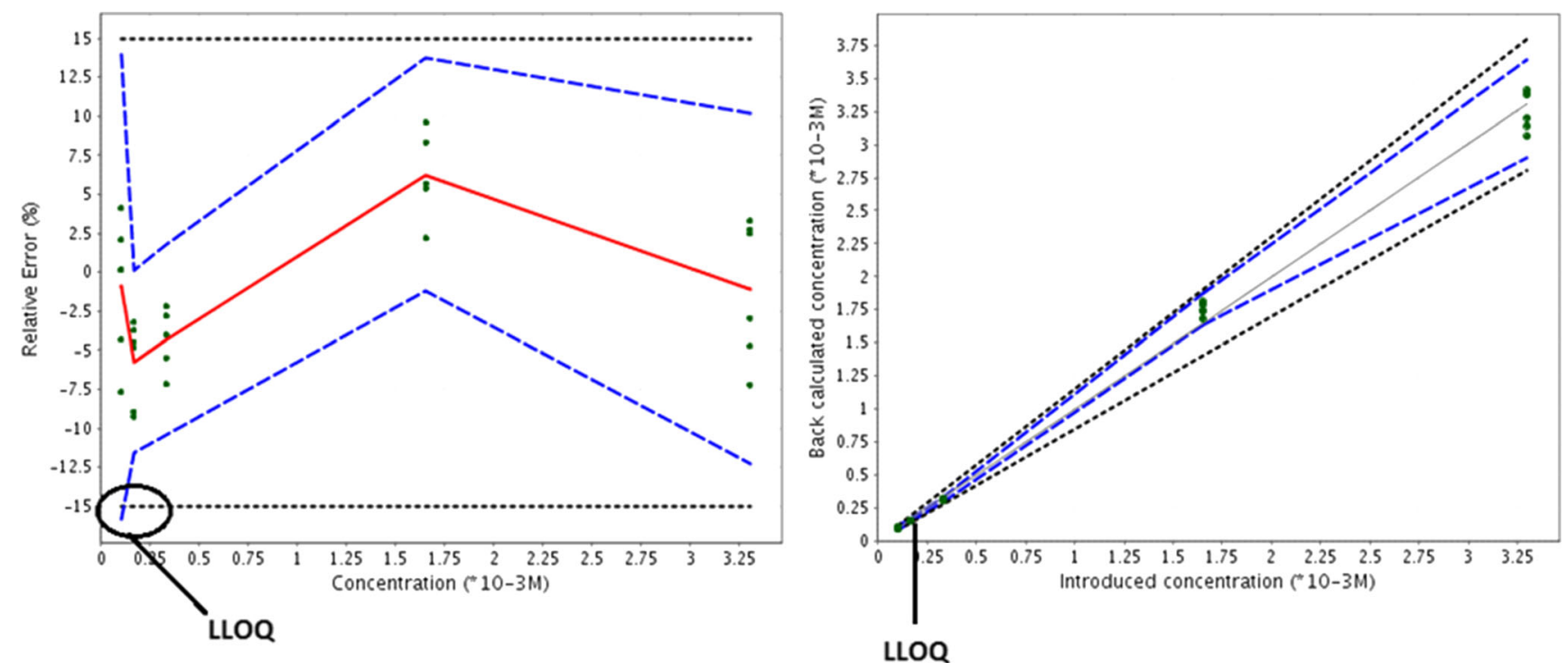

Fig. 4. Left: accuracy profile obtained by considering linear regression after square root transformation. Relative errors of the back-calculated concentrations, represented by green dots, are spread around the relative bias (red line) and comprised of somewhere between the betaexpectation tolerance limits, represented by dashed lines (LLOQ $0.1116 \mathrm{mM}$ ). The dotted lines represent acceptance limits. Right: observed estimated plot (linearity profile) obtained by considering linear regression after square root transformation. The plain line is the identity line: $Y=X$. The dashed limits correspond to the accuracy profile, i.e., the $\beta$-expectation tolerance limits expressed in absolute values. The dotted lines represent the acceptance limits. The method is considered as valid within the range for which the dashed curves are within the dotted acceptance limits (LLOQ $0.112 \mathrm{mM}$ )

cyclodextrin in cell culture medium. Indeed, the presence of lipids (coming from serum additives) impairs the use of $1 \mathrm{D}$ NMR. 2D-COSY NMR could therefore be used in various in vitro experiments such as permeability studies through a cell layer.

\section{DISCUSSION}

Even if lower error estimates have been reported with previously described $1 \mathrm{D}{ }^{1} \mathrm{H}-\mathrm{NMR}$ study (27), overlapping observed in our samples considerably increases technical
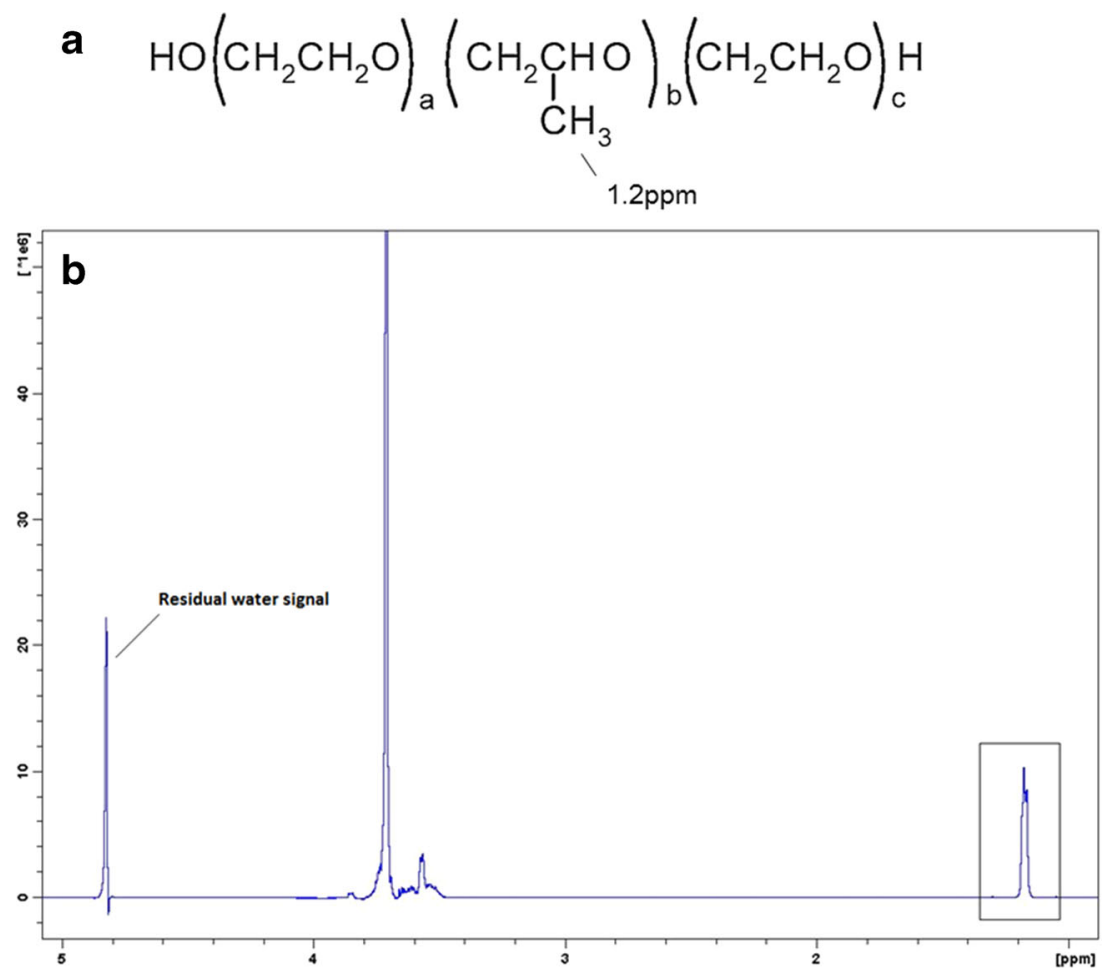

Fig. 5. Chemical structure of poloxamer (ethylene oxide/propylene oxide block copolymer) (a) ${ }^{1} \mathrm{H}-\mathrm{NMR}$ spectrum of water solution spiked with poloxamer 188 (b). The methyl signal at $1.2 \mathrm{ppm}$ is surrounded 


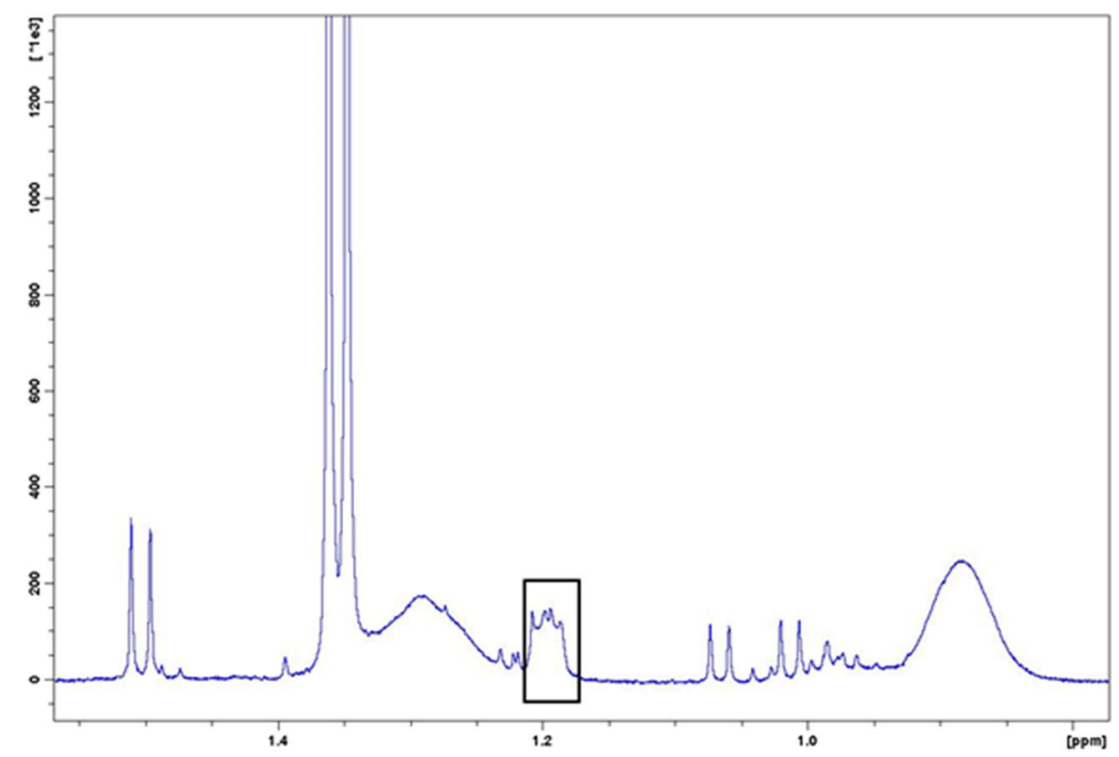

Fig. 6. ${ }^{1} \mathrm{H}-\mathrm{NMR}$ spectrum of plasma solution spiked with poloxamer 188 . The signal at $1.2 \mathrm{ppm}$ corresponding to the methyl group is surrounded

error and therefore impairs its use. In this study, we highlighted the interest of 2D-COSY NMR spectroscopy for the quantification of hardly detectable pharmaceutical molecules in biological matrices, especially related to its ability to accurately quantify a substance of interest without complicated sample pre-treatments. Cyclodextrins represent ideal candidates for NMR analysis because uncertainty in the molecular weight makes them hardly quantifiable by using traditional techniques such as LC/MS. Moreover, needed limits of quantification in toxicological studies are relatively high given that these compounds have already been highlighted for their low toxicity. Indeed, some toxicological studies were undertaken to evaluate the toxicity of cyclodextrins in both humans and animals. They revealed that natural cyclodextrins such as $\beta$-cyclodextrin can induce toxicity especially following i.v. and subcutaneous administrations. Cyclodextrin derivatives (such as 2-HP- $\beta-C D$ ) appear less toxic. For example, following a 1-week intravenous study at a single dose of $1 \mathrm{~g}$, no adverse effects were reported (33). For that purpose, we decided to limit the needed plasma volume to $100 \mu \mathrm{l}$. This enables the use of small rodents such as mouse for which the total blood volume is only 70 to $80 \mathrm{ml} / \mathrm{kg}$ of body weight. Moreover, in order to take into account the variations in terms of lipid and protein concentrations in plasma, we used two different plasmas during the validation process. The total protein concentrations of both plasmas were calculated based on the Bradford dye-binding method and indicated a higher protein concentration ( two times

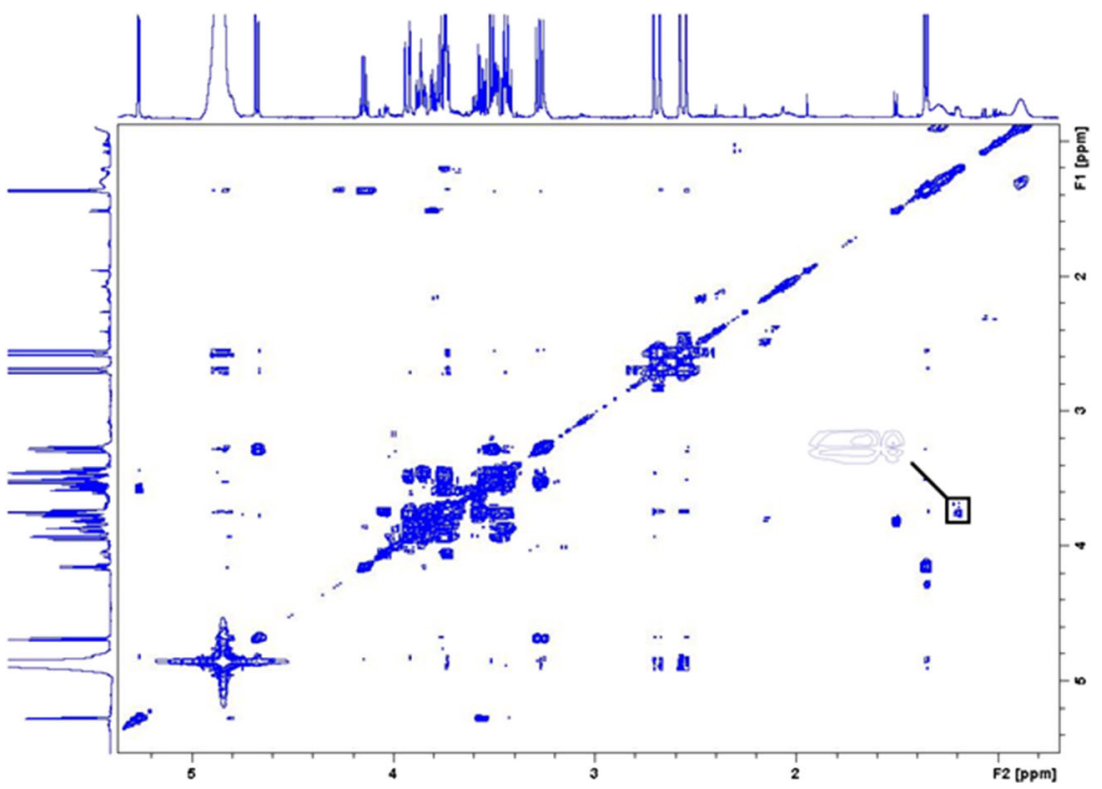

Fig. 7. 2D-COSY NMR spectra of poloxamer 188 in plasma at a concentration of $0.25 \mathrm{mg} / \mathrm{ml}$. The correlation spot between the methyl group $(1.2 \mathrm{ppm})$ and the $\mathrm{CH}_{2} \mathrm{O} / \mathrm{CHO}$ integrals between $\sim 3.6$ and $3.9 \mathrm{ppm}$ is enlarged 
more) in the second plasma. Besides this full validation process, we also analyzed the applicability of this method to other hardly detectable pharmaceutical compounds. We therefore analyzed plasmas spiked with poloxamers 188 and 407 solutions by using the same approach. Similarly, 2D-NMR quantification could also be applied to other pharmaceutical API or excipients by using the same approach. However, conventional 2D-NMR experiments are affected by long acquisition time due to high number of $\mathrm{t} 1$ increments in order to reach sufficient spectral width and resolution (34). Therefore, the lack of sensitivity meets with reasonable acquisition time limit its applicability. In recent years, several strategies have been developed in order to decrease experiment durations including the decrease of recovery delay between two successive $\mathrm{t} 1$ increments. Furthermore, a new approach based on one scan acquisition appears to be of particular interest as a powerful analytical tool. This new approach, called ultra-fast NMR, is continuously growing over years and could also be applied to the specific detection and quantification of pharmaceutical compounds in biological matrices by using the approach based on calibration curves instead of direct quantification. These new sequences could allow a sensitive and accurate quantification without the need of complex sample pre-treatments and could therefore be applied to numerous pharmaceutical developments in a near future.

\section{CONCLUSIONS}

Identification and specific quantification of pharmaceutical compounds in complex biological media is a key challenge in drug development. Classical analytical methods require timeconsuming sample pre-treatments and extractions which could induce bias due to multiple experimenter manipulations. NMR analysis does not require sample treatments or extractions which therefore increases its accuracy in quantification. Quantitative NMR approaches are traditionally based on $1 \mathrm{D}{ }^{1} \mathrm{H}$ NMR spectra which impair their applicability to spectra with signal overlaps as it can be observed in pharmaceutical formulations or in biological matrices. 2D-NMR sequences address this overlap issue thanks to the added dimension. However, due to some factors influencing cross-peak intensities, the direct quantitative relation observed between signal intensities and compound concentrations in 1D-NMR analysis could not be extrapolated to 2D-COSY NMR. In this study, we demonstrated that 2D-COSY spectroscopy could be used as a valuable and accurate analytical technique for the quantification of pharmaceutical compounds, including hardly detectable compounds such as cyclodextrins or poloxamers, in complex biological matrices by using a calibration curve approach. Further to ultra-fast sequences development, 2D-NMR could become in a near future a useful analytical technique with numerous pharmaceutical applications regarding active ingredients and excipients quantifications.

\section{ACKNOWLEDGMENTS}

Authors thank Fabrice Krier for critical comments. Authors also thank the "Fond National de la Recherche Scientifique"-FNRS from which Pascal de Tullio is a senior research associate and the Walloon Region of Belgium for the financial support (WB-health call AEROGAL project).

\section{REFERENCES}

1. Bharti SK, Roy R. Quantitative $1 \mathrm{H}$ NMR spectroscopy. TrAC Trends Anal Chem. 2012;35:5-26.

2. Holzgrabe U. Quantitative NMR, spectroscopy in pharmaceutical applications. Prog Nucl Magn Reson Spectrosc. 2010;57(2):229-40.

3. Simmler C, Napolitano JG, McAlpine JB, Chen SN, Pauli GF. Universal quantitative NMR analysis of complex natural samples. Curr Opin Biotechnol. 2014;25:51-9.

4. Hachem R, Malet-Martino M, Gilard V. First identification and quantification of lorcaserin in an herbal slimming dietary supplement. J Pharm Biomed Anal. 2014;98:94-9.

5. Holzgrabe U, Malet-Martino M. NMR spectroscopy in pharmaceutical and biomedical analysis. J Pharm Biomed Anal. 2014;93:1-2.

6. Pauli GF, Godecke T, Jaki BU, Lankin DC. Quantitative $1 \mathrm{H}$ NMR. Development and potential of an analytical method: an update. J Nat Prod. 2012;75(4):834-51.

7. Holzgrabe U, Deubner R, Schollmayer C, Waibel B. Quantitative NMR spectroscopy-applications in drug analysis. J Pharm Biomed Anal. 2005;38(5):806-12.

8. Lewis IAS, Schommer SC, Hodis B, Robb KA, Tonelli M, Westler WM, et al. Method for determining molar concentrations of metabolites in complex solutions from two-dimensional $1 \mathrm{H}-$ 13C NMR spectra. Anal Chem. 2007;79:6.

9. Giraudeau P. Quantitative 2D liquid-state NMR. Magn Reson Chem. 2014;52(6):259-72.

10. Bingol K, Zhang F, Bruschweiler-Li L, Bruschweiler R. Quantitative analysis of metabolic mixtures by two-dimensional $13 \mathrm{C}$ constant-time TOCSY NMR spectroscopy. Anal Chem. 2013;85(13):6414-20.

11. Gronwald MSK, Kaspar H, Fagerer SR, Nurnberger N, Dettmer $\mathrm{K}$, Bertsch T, et al. Urinary metabolite quantification employing 2D NMR spectroscopy. Anal Chem. 2008;80:10.

12. $\mathrm{Hu} \mathrm{F}$, Furihata K, Kato Y, Tanokura MJ. Nondestructive quantification of organic compounds in whole milk without pretreatment by two-dimensional NMR spectroscopy. J Agric Food Chem. 2007;55:5.

13. Jiang H, Sidhu R, Fujiwara H, De Meulder M, de Vries R, Gong $\mathrm{Y}$, et al. Development and validation of sensitive LC-MS/MS assays for quantification of 2-hydroxypropyl- $\beta$-cyclodextrin in human plasma and CSF. J Lipid Res. 2014;55(7):12.

14. Szeman J, Gerloczy A, Csabai K, Szejtli J, Kis GL, Su P, et al. High-performance liquid chromatographic determination of 2 hydroxypropyl-g-cyclodextrin in different biological fluids based on cyclodextrin enhanced fluorescence. J Chromatogr B. 2002;774:8.

15. Soga M, Ishitsuka Y, Hamasaki M, Yoneda K, Furuya H, Matsuo $M$, et al. HPGCD Outperforms HPBCD as A Potential Treatment for Niemann-Pick Disease Type C During disease Modeling with iPS Cells. Stem cells. 2014.

16. Liu B, Turley SD, Burns DK, Miller AM, Repa JJ, Dietschy JM. Reversal of defective lysosomal transport in NPC disease ameliorates liver dysfunction and neurodegeneration in the npc1-/- mouse. Proc Natl Acad Sci U S A. 2009;106(7):2377-82.

17. Brewster ME, Loftsson T. Cyclodextrins as pharmaceutical solubilizers. Adv Drug Deliv Rev. 2007;59(7):645-66.

18. Loftsson T, Brewster ME. Pharmaceutical applications of cyclodextrins: basic science and product development. J Pharm Pharmacol. 2010;62(11):1607-21.

19. Del Valle EMM. Cyclodextrins and their uses: a review. Process Biochem. 2004:39(9):1033-46.

20. Camargo F, Erickson RP, Garver WS, Hossain GS, Carbone PN, Heidenreich RA, et al. Cyclodextrins in the treatment of a mouse model of Niemann-Pick C disease. Life Sci. 2001;70(2):131-42.

21. Batrakova EV, Kabanov AV. Pluronic block copolymers: evolution of drug delivery concept from inert nanocarriers to biological response modifiers. J Control Release : Off J Control Release Soc. 2008;130(2):98-106.

22. Kabanov AV, Batrakova EV, Sriadibhatla S, Yang Z, Kelly DL, Alakov VY. Polymer genomics: shifting the gene and drug delivery paradigms. J Control Release : Off J Control Release Soc. 2005;101(1-3):259-71. 
23. Chylla RA, Hu K, Ellinger JJ, Markley JL. Deconvolution of two-dimensional NMR spectra by fast maximum likelihood reconstruction: application to quantitative metabolomics. Anal Chem. 2011;83(12):4871-80.

24. Boulanger B, Rozet E, Moonen F, Rudaz S, Hubert P. A riskbased analysis of the AAPS conference report on quantitative bioanalytical methods validation and implementation. J Chromatogr B Anal Technol Biomed Life Sci. 2009;877(23):2235-43.

25. Hubert P, Nguyen-Huu JJ, Boulanger B, Chapuzet E, Chiap P, Cohen $\mathrm{N}$, et al. Harmonization of strategies for the validation of quantitative analytical procedures. A SFSTP proposal-part I. J Pharm Biomed Anal. 2004;36(3):579-86.

26. Rozet E, Marini RD, Ziemons E, Boulanger B, Hubert P. Advances in validation, risk and uncertainty assessment of bioanalytical methods. J Pharm Biomed Anal. 2011;55(4):848-58.

27. Dufour G, Evrard B, de Tullio P. Rapid quantification of 2hydroxypropyl-beta-cyclodextrin in liquid pharmaceutical formulations by $1 \mathrm{H}$ nuclear magnetic resonance spectroscopy. Eur J Pharm Sci : Off J Eur Fed Pharm Sci. 2015;73:20-8.

28. International Conference on Harmonization (ICH) of Technical Requirements for Registration of Pharmaceuticals for Human Use TQRVoAPTaM, Geneva. International Conference on Harmonization (ICH) of Technical Requirements for Registration of Pharmaceuticals for Human Use, Topic Q2 (R1):
Validation of Analytical Procedures: Text and Methodology, Geneva. 2005.

29. Rozet E, Ceccato A, Hubert C, Ziemons E, Oprean R, Rudaz S, et al. Using tolerance intervals in pre-study validation of analytical methods to predict in-study results. J Chromatogr A. 2007;1158:111-25.

30. Mao Y, Thompson MJ, Wang Q, Tsai EW. Quantitation of poloxamers in pharmaceutical formulations using size exclusion chromatography and colorimetric methods. J Pharm Biomed Anal. 2004;35(5):1127-42.

31. Nair LM, Konkel J, Thomas M, Koberda M. Comparison of electrospray ionization mass spectrometry and evaporative light scattering detections for the determination of Poloxamer 188 in itraconazole injectable formulation. J Pharm Biomed Anal. 2006;41(3):725-30.

32. Urban-Morlan Z, Castro-Rios R, Chavez-Montes A, MelgozaContreras LM, Pinon-Segundo E, Ganem-Quintanar A, et al. Determination of poloxamer 188 and poloxamer 407 using highperformance thin-layer chromatography in pharmaceutical formulations. J Pharm Biomed Anal. 2008;46(4):799-803.

33. Gould S, Scott RC. 2-Hydroxypropyl-beta-cyclodextrin (HPbeta-CD): a toxicology review. Food Chem Toxicol : Int J Publ Br Ind Biol Res Assoc. 2005;43(10):1451-9.

34. Giraudeau P, Frydman L. Ultrafast 2D NMR: an emerging tool in analytical spectroscopy. Annu Rev Anal Chem. 2014;7:129-61. 